\title{
SIMULATING NONUNIFORM PROPERTIES IN POLYMER-ELECTROLYTE FUEL CELLS
}

\begin{abstract}
A. Z. Weber and J. Newman
Lawrence Berkeley National Laboratory, Berkeley, CA 94720, USA

In this transaction, results from mathematical, pseudo 2-D simulations are shown for four different thickness distributions of both the membrane and cathode catalyst layer. The results and subsequent analysis clearly demonstrate that the position along the gas channel is more important than the local thickness variations, especially for the membrane.
\end{abstract}

\section{Modeling Methodology and Approach}

Mathematical modeling and simulation is ideally suited to examining local effects and properties that are not accessible experimentally. One such issue is how the local variation in layer thickness impacts performance. It is known that due to manufacturing and production processes, it is hard to obtain uniform material properties, especially thickness. While some thickness variation may be acceptable, the limits are not known. In this transaction, we examine the effect of local variations in the membrane and catalyst-layer thicknesses on both the local and global performance. Three distributions for each thickness are used as determined from SEM micrographs and shown in Figure 1. A fourth distribution is that of uniform thickness (the solid lines in the figure) with values of 13.5 and $30 \mu \mathrm{m}$ for the catalyst layer and membrane, respectively. In terms of the distributions, the gas channel was discretized into 32 segments, with every two segments having the same thickness. The distributions clearly show that there is a great deal of variability in the local thickness values; however, the average values for the different distributions are very similar.
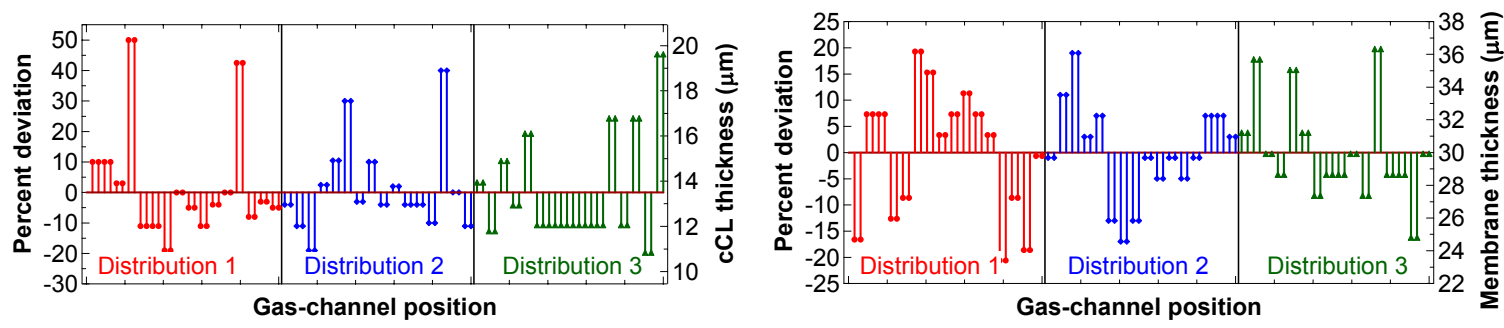

Figure 1. Distributions for the catalyst-layer (left) and membrane (right) thicknesses.

Simulations are conducted using a pseudo 2-D approach, where a 1-D cell-sandwich model is run at various segments along the gas channel. ${ }^{1}$ The thickness of either the membrane or cathode catalyst layer is set to a different value in each segment as determined by the distributions shown in Figure 1. The 1-D sandwich is composed of symmetric diffusion media, anode and cathode catalyst layers, and membrane. The model is based on our previous work ${ }^{2}$ and accounts for liquid and vapor flow throughout all of the fuel-cell sandwich layers, nonisothermal phenomena, and electrochemical kinetics and phenomena. For the pseudo 2-D approach, both coflow and crossflow 
conditions are simulated, although the coflow results are emphasized in this manuscript. Typical operating conditions are $65^{\circ} \mathrm{C}$ inlet and coolant temperature, ambient pressure, 1.2 and 2.0 hydrogen and air stoichiometry, respectively, and $0.6 \mathrm{~V}$.

\section{$\underline{\text { Results and Discussion }}$}

Before proceeding to examine the effects of the local variations, it is of interest to examine how performance is affected by changes in the membrane and catalyst-layer thicknesses. To this end, simulations are undergone where the thickness was changed uniformly to different values. The resulting impact on performance is shown in Figure 2.

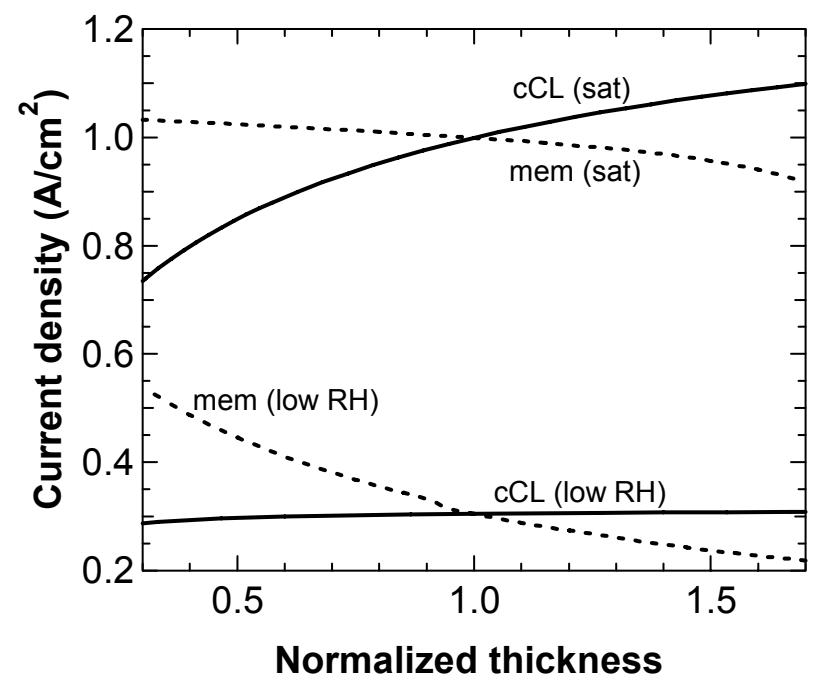

Figure 2. Impact of catalyst-layer and membrane thickness on the overall current density. The thickness is uniform along the gas channel and is normalized to the average thickness of the respective layer. The two sets of line indicate saturated and $25 \% \mathrm{RH}$ feed streams.

Figure 2 shows the expected results that at $0.6 \mathrm{~V}$, the thinner the membrane the better and the thicker the cathode catalyst layer the better. It should be noted that when changing the cathode catalyst-layer thickness, all other parameters are also scaled. Thus, the thicker catalyst layer contains an overall higher loading of platinum. This assumption is used since it seems to agree with the micrographs and also in the distribution studies later, where the average loading is similar for the distributions but the local value can vary. Because of the above aspect, the catalyst layer does not demonstrate a maximum (although it will at lower potentials) as one might expect due to the additional masstransport limitations inherent in a thicker layer. Practically, the membrane thickness cannot be too small or pinhole formation and gas crossover will become problematic (this occurs at much smaller thicknesses than those shown in Figure 2). Similarly, the catalyst layer cannot become too thick due to the economic considerations of platinum.

The curves in Figure 2 demonstrate that the performance is a stronger function of catalyst-layer rather than membrane thickness for saturated feeds. This is primarily due to the increased area available for reaction with the thicker layer. Furthermore, for the saturated system, the change in membrane thickness only has a minimal impact on performance and system water balance. However, this is not the case for the low relative 
humidity cases, where the inlet gases are both fed at $25 \%$ RH. For these cases, the impact of the membrane thickness on the water balance and water management has a greater influence than changing the catalyst-layer thickness. This is because the system is not as reaction limited as for the saturated case, and humidification of the anode stream, in particular, is important for increased performance.

Figure 2 displays the overall impact of performance, but the question remains how this correlates to the local changes due to the local thickness variability. First, we examine the membrane thickness and then the catalyst-layer changes. Figure 3 shows the impact that the membrane-thickness distributions have on both the local temperature and current density for both the saturated and low-relative-humidity cases (the cathodecatalyst-layer thickness is uniform at $13.5 \mu \mathrm{m}$ ).
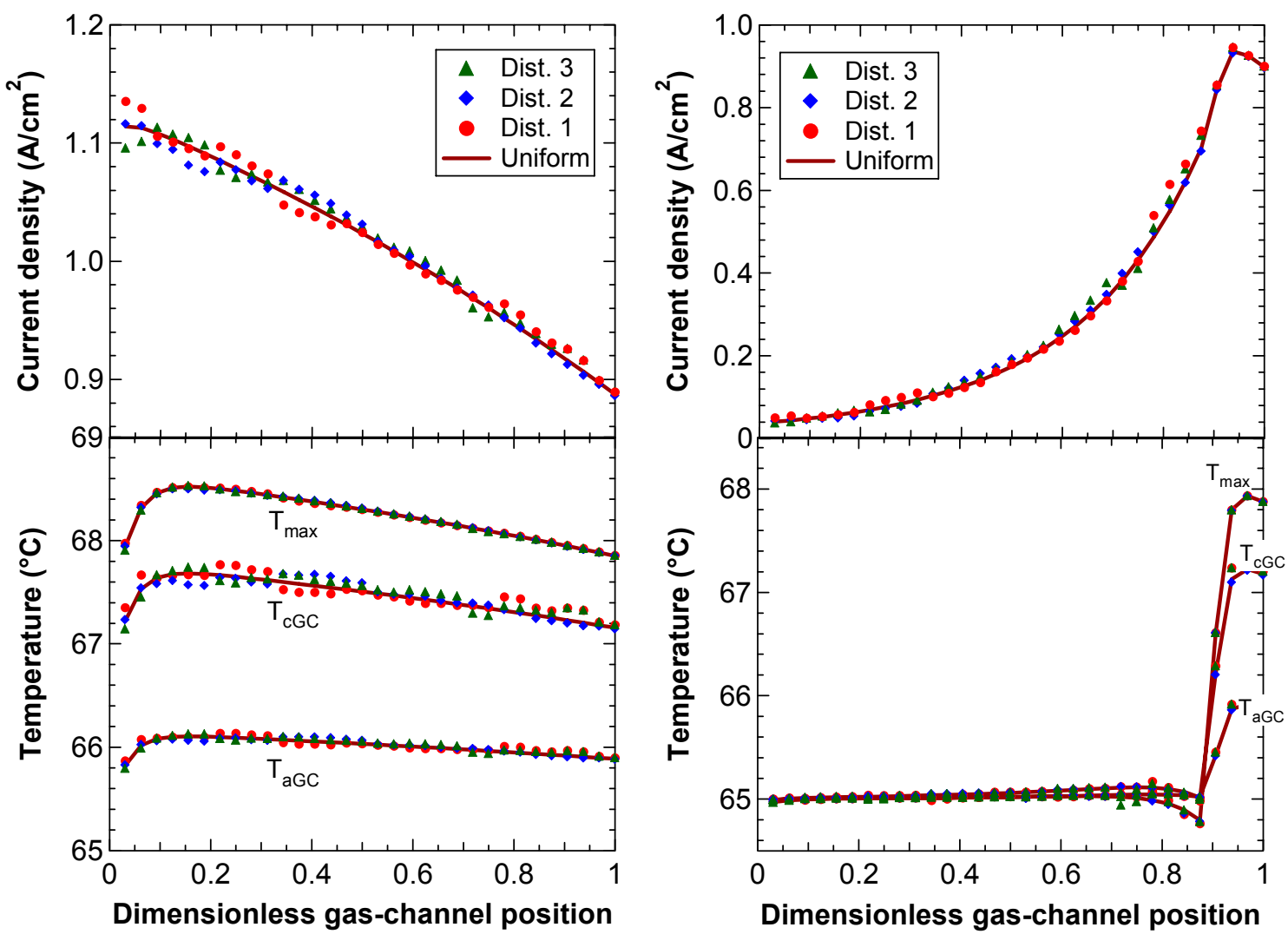

Figure 3. Along-the-channel values for the current density and maximum and anode- and cathode-gas-channel temperatures for both saturated (left) and 25\% RH (right) feeds at $0.6 \mathrm{~V}$ as a function of membrane thickness distribution. The distributions correspond to those shown in Figure 1, and the solid line indicates a uniform thickness at the average value of $30 \mu \mathrm{m}$.

From the figure, it is clear that the results seen in Figure 2 hold in that the local variations in membrane thickness do not impact performance significantly. This is true even for the low RH case, where one might have expected to see a greater dependence on the thickness given the results in Figure 2. However, the gas-channel position is more dominant than the local thickness in determining performance. For the low RH case, the performance is mainly dictated by the humidification increase along the channel. For the 
saturated case, the consumption of oxygen is the main driver of the shape of the current distribution.

Of perhaps more importance from a durability standpoint is the temperature distribution along the channel. As seen in Figure 3, and in accordance with the currentdensity distributions, the temperature distributions are also dominated by channel position and not the local thickness. This is especially true for the maximum temperature (which occurs in the cathode catalyst layer) and the anode-gas-channel temperature. The cathode-gas-channel temperature has the largest deviations due to the way in which the energy balance is affected by evaporation and condensation and the water balance as well as the heat conduction through the membrane. For the saturated case, the temperatures increase near the inlet because of the lack of a sufficient heat flux to the coolant due to the low temperature difference, and then the temperatures decrease because of the smaller heat generation due to the lower current density. Figure 3 also shows that there are temperature gradients of a couple of degrees within the cell sandwich; the average cell temperature is also a few degrees above that of the coolant stream. For the low-relativehumidity case, the temperature distribution is very different and the effect of the local membrane variations much smaller. In fact, until a liquid-water phase forms, there is no appreciable temperature difference in the cell sandwich from that of the coolant, i.e., the cell is isothermal. This is interesting since the current-density distribution is highly nonuniform, and it underscores the point that water phase changes comprise very large heat sources and sinks. This last point is underscored in the way that the temperature changes once liquid water is formed near the outlet in Figure 3.

Similar to Figure 3, Figure 4 examines the local effect of the cathode-catalyst-layer thickness distributions (the membrane thickness is taken to be a uniform $30 \mu \mathrm{m}$ ). As expected from Figure 2, for the low-relative-humidity case there is almost no effect of changing the layer thickness on the current density or temperature, until liquid water is formed, similar to the results shown in Figure 3. Also as expected from Figure 2, there is a much stronger effect of local cathode-catalyst-layer thickness variations on performance for the saturated case, as well as a stronger dependence than that seen for the membrane-thickness variations. If one normalizes the results to the uniform case, one sees a strong correlation between performance and thickness deviation with slopes similar to those in Figure 2 for cathode-catalyst-layer thickness-distribution results. However, a similar analysis with the membrane-thickness-distribution results in only data scatter and no strong correlation. The comparative analysis clearly demonstrates that the catalyst-layer thickness has a greater impact on the local performance than the membrane thickness, above and beyond the fact that the deviations are slightly larger on a percentage basis for the cathode catalyst layer (see Figure 1). Furthermore, the local performances for the membrane-distribution cases in comparison to the cathode-catalystlayer ones are more dominated by gas-channel location rather than local variations.

The deviations in both current density and especially the maximum temperature for the saturated cases in Figure 4 could be a concern in terms of lifetime and durability. The heterogeneities that they represent demonstrate unequal heating (i.e., hot spots) as well as different reactant utilization and water balance. Such nonuniformities result in concerns of unequal stressing of the various components, with the most important probably being the membrane. While the magnitudes seen in Figure 4 may not be a major concern, 
these nonuniformities will become larger at higher current densities. They may also cause difficulties and accentuate local failure mechanisms during transient operation and cycling conditions. Finally, it should be remarked that at a certain point the deviations may become large enough in magnitude that the pseudo 2-D assumption breaks down, in which case a full 2-D simulation would be required.
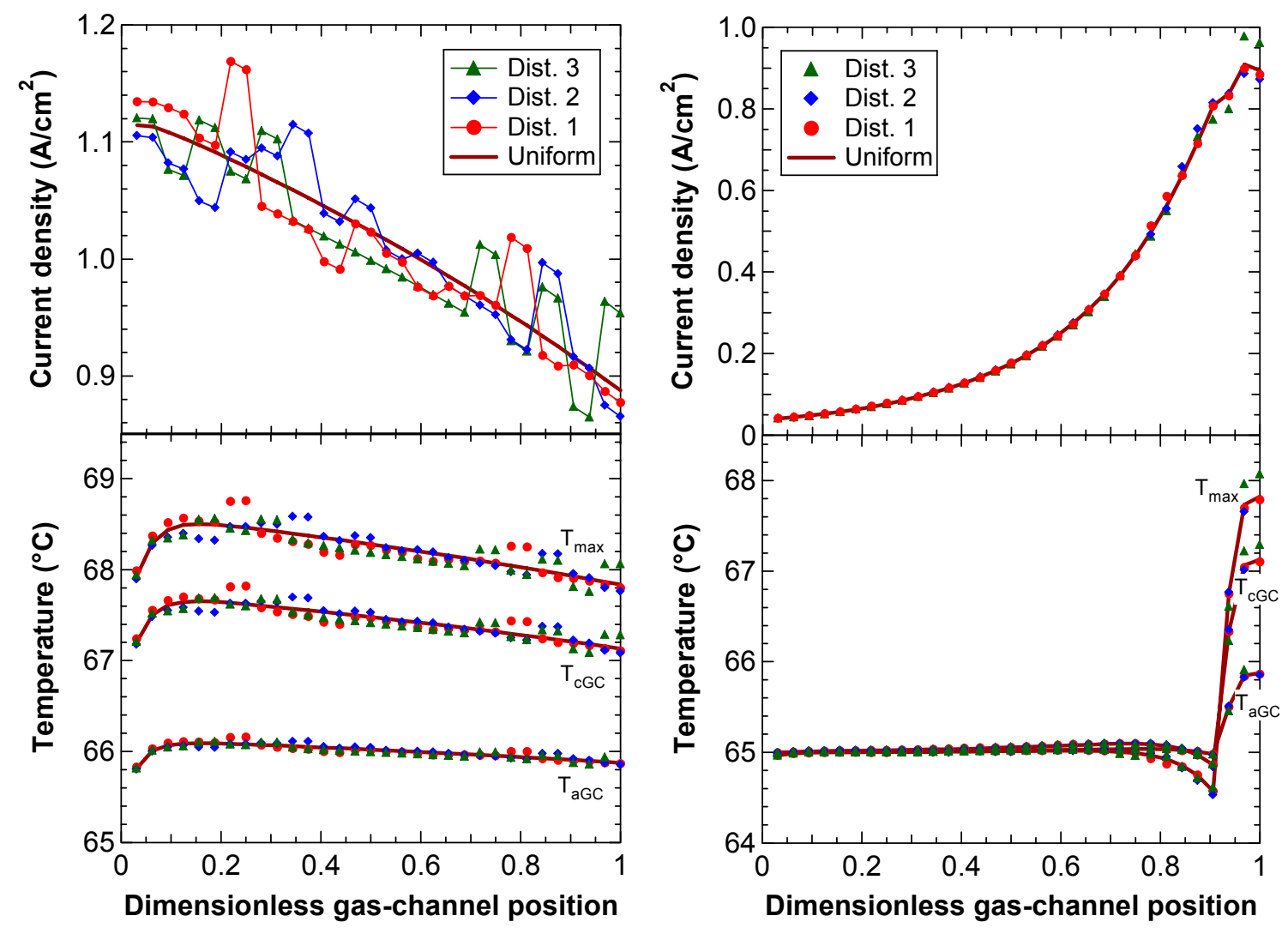

Figure 4. Along-the-channel values for the current density and maximum and anode- and cathode-gas-channel temperatures for both saturated (left) and $25 \% \mathrm{RH}$ (right) feeds at $0.6 \mathrm{~V}$ as a function of cathode-catalyst-layer thickness distribution. The distributions correspond to those shown in Figure 1, and the solid thick line indicates a uniform thickness at the average value of $13.5 \mu \mathrm{m}$.

The above analysis is focused on how the thickness distributions impact the local performance. Before concluding, remarks should be made on how the global performance is impacted. To do this comparison, the deviation from the uniform case of the average value of the current density from Figures 3 and 4 is calculated for each distribution. The results are given in Figure 5. From the figure, it is clear that the deviations for the cathode-catalyst-layer thickness have no appreciable impact on the overall performance. This is not expected since the average thickness is essentially the same as that of the uniform distribution; however, it is also somewhat disconcerting considering that these cases showed the largest local performance variability (see Figure 4). Thus, while the overall performance may look good, there still could be local variations that are problematic. In other words, the global performance cannot be used as a signature to detect local problems and nonuniformities, until probably too late and significant damage has occurred. 


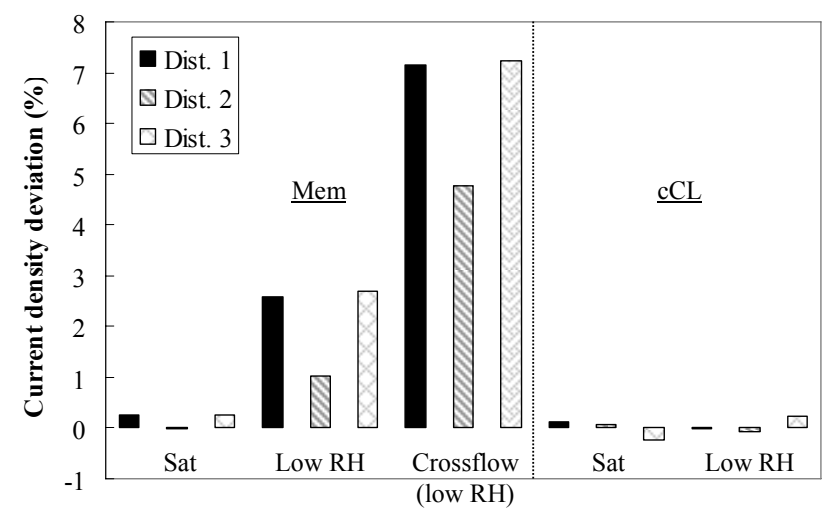

Figure 5. Deviation in the average current density at $0.6 \mathrm{~V}$ as a function of distribution for both the membrane and cathode-catalyst-layer thickness. Included are the saturated and $25 \% \mathrm{RH}$ feed cases as well as a simulation $25 \% \mathrm{RH}$ feed case using a crossflow instead of coflow configuration.

For the membrane cases, the saturated feed conditions do not show a large impact on the global performance. For the low-relative-humidity cases, larger deviations are seen as expected from the impact of membrane thickness on the water balance (see Figure 2) as well as the more nonuniform current-density distribution. This is interesting because it emphasizes the fact that even though the local variations are not too large (see Figure 3) compared to the overall trends, they are significant enough to change the global current density a few percent. This is not true for the cathode-catalyst-layer studies as seen in the exact agreement in the inlet region of the current-density distribution in Figure 4. To assess the impact of flow geometry, the membrane-thickness-distribution cases are run in a crossflow instead of coflow arrangement. In such a case, air is fed to the top and hydrogen to the side of a 2-D network of 1-D sandwich-layer-model segments. As seen in Figure 5, such a case results in the largest deviations in terms of global performance. These larger deviations are due to the different hydration profile that exists in the crossflow arrangement, and the impact of the membrane thickness on water management. Overall, the deviations on a global scale are largest for the low relative-humidity cases, and one would expect similar variability of around $5 \%$ or so in experimental data that would be directly due to manufacturing nonuniformity in the various layer thicknesses. Finally, although not shown, similar studies where both layer thicknesses distributions are considered show an almost additive effect under the above simulation conditions.

\section{Summary}

Simulations with various distributions determined from experimental data of either membrane or cathode-catalyst-layer thickness were accomplished. Results indicated that having a nonuniform membrane thickness only marginal affected the local performance; the location of a point along the gas channel was more important than the actual deviation in membrane thickness. Globally, the distributions resulted in at most a few percentage change in the average current density, with larger deviations with low-relative-humidity feeds due to the importance of the membrane thickness in fuel-cell water management. The cathode-catalyst-layer thickness distribution resulted in more local variations than for 
the membrane case. These deviations could result in problems in terms of durability, and were larger for saturated conditions.

\section{$\underline{\text { Acknowledgements }}$}

This work was supported by the Assistant Secretary for Energy Efficiency and Renewable Energy, Office of Hydrogen, Fuel Cell, and Infrastructure Technologies, of the U. S. Department of Energy under contract number DE-AC02-05CH11231. Plug Power is acknowledged for providing the experimental distribution data.

\section{$\underline{\text { References }}$}

1. A. Z. Weber and J. Newman, Chemical Reviews, 104, 4679 (2004).

2. A. Z. Weber and J. Newman, J. Electrochem. Soc., in press (2006). 